\title{
A pilot study of vasculogenic mimicry immunohistochemical expression in intraocular melanoma model
}

\author{
LUXIA CHEN $^{1 *}$, SHIWU ZHANG $^{2 *}$, XIAORONG LI $^{1}$, BAOCUN SUN $^{2,3}$, \\ XIULAN ZHAO ${ }^{3}$, DANFANG ZHANG ${ }^{2}$ and SHAOZHEN ZHAO ${ }^{1}$ \\ ${ }^{1}$ Eye Center of Tianjin Medical University, Tianjin 300070; ${ }^{2}$ Department of Pathology, Tianjin Cancer \\ Hospital; ${ }^{3}$ Department of Pathology, Tianjin Medical University, Tianjin 300060, P.R. China
}

Received October 15, 2008; Accepted December 9, 2008

DOI: 10.3892/or_00000313

\begin{abstract}
Vasculogenic mimicry (VM) has been recognized as a new form of angiogenesis. However, some previous studies have demonstrated the absence of VM channel in a uveal melanoma xenograft mice model. This study investigated the pattern and distribution of microcirculation in an intraocular animal model. C57Bl/6 mice were randomly divided into 3 groups used for the blood supply models of malignant melanoma. The right eyes of the mice received subretinal injections with B16 melanoma cells and the left eyes were the control. One experimental group mice was randomly sacrificed at days 3, 7 and 14 to evaluate the tumor size and microcirculation by immunostaining with anti-CD34 antibodies, PAS staining and electronic microscopy (EM). Activatedcarbon tracing was used to confirm whether the VM structure connected to the host blood circulation at day 14 . We observed 3 types of microcirculation patterns in this model. The tracer was used to confirm whether VM structure connected to the host blood circulation. The distribution of $\mathrm{VM}$ and MV was not uniform and appeared in patches. As the area of tumor tissue expands, the number of endothelium increases and that of VM decreases. The number of endothelium-dependent vessels correlated with the tumor size $(r=0.805, P=0.000)$, while the number of $\mathrm{VM}$ was inversely correlated $(\mathrm{r}=0.47, \mathrm{P}=0.03)$. The EM results validated the presence of 3 patterns. In conclusion, VM along with endothelium-dependent vessels and MV sustained the blood supply. Tumor cells can obtain oxygen and nutriment through VM and MV besides endothelium-dependent vessels. VM may be a way to adapt to rapid tumor growth and invasiveness.
\end{abstract}

Correspondence to: Dr Baocun Sun, Tianjin Cancer Hospital, Tianjin Medical University, Tianjin 300060, P.R. China

E-mail: lq1012@yahoo.com.cn

${ }^{*}$ Contributed equally

Key words: melanoma, vasculogenic mimicry, animal model, microcirculation patterns

\section{Introduction}

Melanoma is a tumor with a high incidence of metastasis and a high mortality rate, since a few mm-thick tumor has a full potential to kill the host in $>80 \%$ of the cases. The microcirculation of primary melanomas, their precursors, and their metastases is distinctive. It is now known that the generation of this patterning is genetically dysregulated in melanoma cells. The concept of 'vasculogenic mimicry' was introduced to describe the unique ability of highly aggressive tumor cells to form capillary-like structure and matrix-rich patterned network in three-dimensional cultures that mimic embryonic vasculogenic network (1). Based on tissue structure and function, VM is different from the endothelial-dependent vessel and mosaic vessel (2). The characteristics of VM areas are that there is no endothelial cell lining the wall of VM but tumor cells imitate the functions of the endothelium, forming the wall of vessels with red blood cells inside it. This study verified the presence of the three types of patterns in intraocular melanoma in C57B1/6 mice and investigated their morphologic structure and their distribution.

\section{Materials and methods}

All experiments followed the guidelines contained within the ARVO statement for the use of animals in ophthalmic and vision research.

Cells. A single cell suspension of B16 malignant melanoma was provided by the Tianjin Cancer Hospital Department of Biochemistry and stored in liquid nitrogen. Before injection, the suspension was incubated for $20-30 \mathrm{sec}$ in a $43^{\circ} \mathrm{C}$ water bath and centrifuged at 1000 gps for $10 \mathrm{~min}$. The supernatant was absorbed by an aseptic tampon, and the pellet was diluted with $0.25 \mathrm{ml} 0.9 \% \mathrm{NaCl}$ solution to ensure a final cell density of $4 \times 10^{7}$ cells $/ \mathrm{ml}$.

Tumor-bearing animal models. The $30 \mathrm{C} 57 \mathrm{Bl} / 6$ mice used were black, female, 6-8 weeks old and were supplied by the Experimental Animal Institute of the Chinese Academy of Medical Science. The average weight of these mice was $21 \mathrm{~g}$ (20-25g). The mice were fed in a temperature-controlled room for 1 week prior to use. All $30 \mathrm{C} 57 \mathrm{~B} 1 / 6$ mice were divided randomly into 3 groups (10 mice per group). A fresh 
suspension of B16 cells line $\left(10^{7}\right.$ cells per mouse) was injected into one eye of the animal (orthotopic injection). Before tumor implantation, all C57B1/6 mice were anesthetized with a mixture of $5 \%$ chloral hydrate $(500 \mathrm{mg} / \mathrm{kg}$ body weight). A drop of $0.5 \%$ oxybuprocaine hydrochloride was applied on the cornea. The right eye of 30 mice received microscopically guided subretinal injections of $0.004 \mathrm{ml}$ of B16 suspension containing $4 \times 10^{7}$ B16 cells $/ \mathrm{ml}$. The left eye of each animal was not injected and served as a control.

After anesthesia, the pupil was dilated with $0.5 \%$ tropicamide and the animal was placed under a Topcon OMS 110 operating biomicroscope. A drop of $2 \%$ methylcellulose was applied on the cornea and a microscope cover slip placed on top of the methylcellulose drop. Thus, the fundus of the mouse eye could be easily observed through the dissecting microscope. Subsequently, the eye was fixed in this position with forceps. A 0.5-cc insulin syringe with an attached 28.5-gauge needle was then inserted into the orbit behind the eye and gently advanced transclerally toward the retina until the level $\left(\sim 15^{\circ} \mathrm{C}\right)$ of the needle tip was visible just underneath the level of the retinal vessels. Then a $10 \mu 1$ microinjector was inserted into the subretinal space (4). Special care was taken to inject the cell suspension in the subretinal space and not into the vitreous or orbit. The mice were checked daily for signs of inflammation in the injected eye by slip-lamp biomicroscopy and weekly by fundus examination using the method described above. Fourteen days after inoculation, a tumor mass with an approximate size of $500-900 \mathrm{~mm}^{3}$ was observed in the site of injection. At days 3, 7 and 14, one group of 10 mice was randomly sacrificed.

Activated-carbon tracing. Fourteen days after B16 cells were inoculated into $\mathrm{C} 57 \mathrm{Bl} / 6$ mice, activated carbon was injected into the mice through the caudal veins. The mice were sacrificed 20 min later and the tumor masses were removed followed by routine fixing, embedding and sectioning. Briefly, the sections were deparaffinized with xylene and rehydrated in graded ethanol solutions (100-75\%). The sections were then put into a solution of picric in ethanol (95\%) for $30 \mathrm{~min}$ and then washed with clear water for $10 \mathrm{~min}$. The results using control sections indicated that formalin granules could be removed from the paraffin-embedded sections using this method. After removal of the formalin granules, the sections were immersed in an acid-potassium permanganate solution for $3 \mathrm{~h}$. After washing with clear water, they were bleached using $2 \%$ oxalic acid solution for $5 \mathrm{~min}$. The results with control sections indicated that the melanin could be removed from these tissue sections by this procedure.

Histochemical and immunohistochemical dual-staining. All tissues in this study were removed, fixed with formalin and embedded in paraffin. The $4-\mu \mathrm{m}$ paraffin-embedded tissue sections were mounted onto poly-L-lysine-coated slides. Paraffin sections were routinely deparaffined. Histochemical staining was performed respectively with hematoxylin-eosin and PAS without hematoxylin counterstaining to facilitate identification of microcirculation patterns. Normal human stomach mucous membrane was the positive control. Immunohistochemical staining was conducted using a two-step method. The antibodies used in this study were anti-CD34 (clone QBEnd/10) purchased from Sigma Chemical Co. (St. Louis, MO, USA), which were used at dilutions of 1:200. Endogenous peroxidase activity was blocked with $3 \%$ hydrogen peroxide in $50 \%$ methanol for $10 \mathrm{~min}$ at room temperature. The sections were rehydrated and washed with PBS and then pretreated with citrate buffer $(0.01 \mathrm{M}$ citric acid, $\mathrm{pH}$ 6.0) for $20 \mathrm{~min}$ at $100^{\circ} \mathrm{C}$ in a microwave oven. Nonspecific binding sites were blocked by exposure to $2 \%$ normal goat serum in phosphate-buffered saline (PBS) for $20 \mathrm{~min}$ at $37^{\circ} \mathrm{C}$. The sections were then incubated overnight at $4^{\circ} \mathrm{C}$ with antiCD34.

After treatment, the sections were rinsed with PBS, incubated with biotinylated goat anti-mouse $\operatorname{IgG}$ for $20 \mathrm{~min}$ at $37^{\circ} \mathrm{C}$, incubated with $3,3^{\prime}$-diaminobenzidine chromogen for $10 \mathrm{~min}$ at room temperature, and washed with distilled water. Then, the sections were rinsed with clear water for $1 \mathrm{~min}$ in order to stop the DAB-staining reaction. Formalin and melanin granules were then removed using the methods mentioned above. Finally, the sections were rinsed with distilled water for $5 \mathrm{~min}$ and incubated with PAS for $15 \mathrm{~min}$. All of the sections were counterstained with hematoxylin, dehydrated in a graded ethanol solutions (75-100\%), cleared with xylene, and mounted with neutrogum.

VM channels and endothelium-dependent vessels quantification. The sections stained with PAS were viewed at $\mathrm{x} 400$, and 10 fields were chosen randomly. The channels defined as VM were enclosed by melanoma cells and lined by PASpositive material with red cells in the center of the channels. The average number of VM channel and endotheliumdependent vessels was determined by our previously demonstrated method. According to the standards introduced (9), capillary vessels and microvessels in the tumor stained with CD34 were counted. A single positively stained endothelial cell can be counted as one MVD, when the area of a vessel is large (>8 red blood cells).

Electron microscopy. Specimens from the intraocular tumor tissues were randomly selected and fixed in $4 \%$ buffered glutaraldehyde and then postfixed in $1 \%$ osmium tetroxide. After dehydration in ethanol and propylene, cells were embedded in Epon epoxy resin. The presence of red blood cells in the channels without endothelium was used as a criterion for authentic VM. The presence of red blood cells in the channels with endothelium and tumor cells was used as a criterion for an authentic mosaic vessel. Ultra-thin sections stained with uranyl acetate and lead citrate were examined with a JEM-1010 transmission electron microscope.

\section{Results}

Results from the intraocular melanoma animal model. All 30 eyes that received a subretinal space B16 cell suspension developed intraocular tumors. Clinically, a consistent pattern of growth was observed: 1 week after injection a mass was observed to have been formed subretinally in the mouse eye by fundus examination. Also in most mice a slight protrusion of the eyeball was noted. Two weeks after injection the subretinal mass had enlarged considerably and a more or less pronounced proptosis was visible in all animals (Fig. 1A). 
Table I. The average number of vasculogenic mimicry and endothelium-dependent vessels varied at different times.

\begin{tabular}{lccc}
\hline \multirow{4}{*}{ Day } \\
\cline { 2 - 4 } Group & 3 & 7 & 14 \\
\hline TV & $51.63 \pm 2.50$ & $112.25 \pm 3.99$ & $750.50 \pm 22.70$ \\
VM & $7.18 \pm 1.54$ & $6.92 \pm 2.26$ & $6.03 \pm 1.86$ \\
E & $0.09 \pm 0.032$ & $3.63 \pm 0.40$ & $5.56 \pm 0.96$ \\
\hline
\end{tabular}

$\mathrm{TV}$, tumor volume; VM, vasculogenic mimicry; E, endotheliumdependent vessels. TV $=(\text { length } \mathrm{x} \text { width })^{2} / 2$

Generally, the B16 melanoma tumors were black with certain blood vessels on their surfaces. The boundary region between the tumor and the surrounding normal tissue was clear. However, certain tumors invaded the adjacent normal tissue. In 21 eyes the tumors were confined to the choroidal space and in the remaining 6 eyes the tumors extended also into the orbit. When the tumor mass was split, the tumor was found to be very soft. The tumor tissues were embedded with paraffin, and sectioned. H\&E sections of the tumors showed three co-existing patterns of blood supply. In the center of the VM, there were numerous red blood cells. Rich blood sinusoids were observed in the sections of melanoma models, and VM with red blood cells were also found (Fig. 1B). Moreover, under an oil microscope, melanin granules were identified in the cytoplasm of tumor cells that formed in the vessels, which indicated that these cells were melanoma cells. Besides VM, MV that consisted of both tumor and vascular endothelial cells was found in the same tumor mass (Fig. 1C). Endotheliumdependent vessels were also observed in this tissue (Fig. 1D).

Analysis of VM channel formation. Counting the microcirculation patterns in the tumors indicated that the expression of VM and endothelium-dependent vessels in different stages of tumor growth has changed. To identify the time pattern of tumor blood supply in different stages of melanoma growth, we measured the density of each type of vessel at day 3, 7 and 14 after inoculation. As the tumor volume [TV = (length $\mathrm{x}$ width) ${ }^{2} / 2$ ] expanded, the number of endothelium-dependent vessels highly correlated with tumor volume $(r=0.805$, $\mathrm{P}=0.000)$, while the number of $\mathrm{VM}$ correlated with tumor volume $(r=0.47, \mathrm{P}=0.03$, Table $\mathrm{I})$.

Results from histochemical and immunohistochemical double staining. PAS staining and CD34 staining were simultaneously performed to confirm the structure of three blood patterns in consecutive sections. CD34 is a marker of endothelial cells, and the base membrane is positive for PAS, therefore CD34 and PAS dual-staining was used to distinguish VM and endothelial-dependent vessels. The VM channels with walls negative for CD34 confirmed that the channels were not composed of endothelial cells. Furthermore, we also observed that certain channels were lined with both CD34 and PAS positive, that is, mosaic channels composed of tumor cells and endothelial cells. Due to the sufficient blood supply offered by VM and MV, no necrosis was found (Fig. 1E).
Activated-carbon tracing results. Activated-carbon tracing was used to conform whether the VM structure connected to the host blood circulation after 14 days. Results of H\&E sections of the tumors showed three co-existing patterns of blood supply. In the center of the VM, there were numerous red blood cells and many brown granules, which must be activated carbon because formalin and melanin were removed previously (Fig. 1F).

Transelectron microscopy. VM channels were further validated by electronmicroscopy. When samples were removed from the same tumor and observed under an electronic microscope, the structure of VM and MV were clearly identified and also red blood cells appeared in the middle of the VM (Fig. 2A and B).

\section{Discussion}

Injection of B16 melanoma cells into the subretinal space of C57B1/6 mouse eyes generates a tumor. The eye generates a tumor that recapitulates the internal architecture and microcirculation of aggressive model. However, the mouse eye may provide a more convenient model as there are more probes and antibodies available for mouse cell types than for rat cells. Moreover, it needs no immunoinhibitors and is convenient to raise. Therefore, ocular tumors are well suited for the study of the tumor microcirculation and hematogenous dissemination of cancer.

Tumors have long been known to depend on angiogenesis to develop, rapidly grow, and successfully metastasize. In our study, it is an exceptionally useful model for testing genetic modulation of $\mathrm{VM}$ for the purposes of designing treatments for highly aggressive tumors $(5,6)$. We had identified formation of looping PAS-positive patterns in these tumors. However, certain scholars have challenged the concept of VM as a pattern of blood supply for tumors $(3)$. Ma et al $(7,8)$ have used both human and mouse melanoma cells implanted into the eyes of immunosuppressed mice to study immunological and molecular events in metastasis, but the presence of VM in these models has not been reported. Clarijs et al (9) reported contradictory findings and they have demonstrated the absence of blood conducting extracellular matrix networks in a uveal melanoma xenograft model in mice. However, we found the existence of VM channels in tumor tissues by employing mouse models bearing B16 malignant melanoma. We found VM with many RBCs in the tube lined with tumor cells. In order to clarify the function of VM, we injected activated carbons via the tail. We subsequently found that activated-carbon granules were observed in the VM and endothelium-dependent vessels in the paraffin-embedded sections. Formalin and melanin were removed from melanoma cells so as not to interfere with actidose detection. This study showed that the tumor tissue connected with host vessels for blood supply by VM. Therefore, we think different tumor cells have different patterns of blood supply in which some can access blood supply by tumor vasculogenesis, and others acquire sufficient oxygen and nutrition by VM (10).

VM is a functional microcirculation for tumor growth, invasion, and metastasis (11). Since it was proposed, it has been challenged. Certain scholars considered VM to be only 

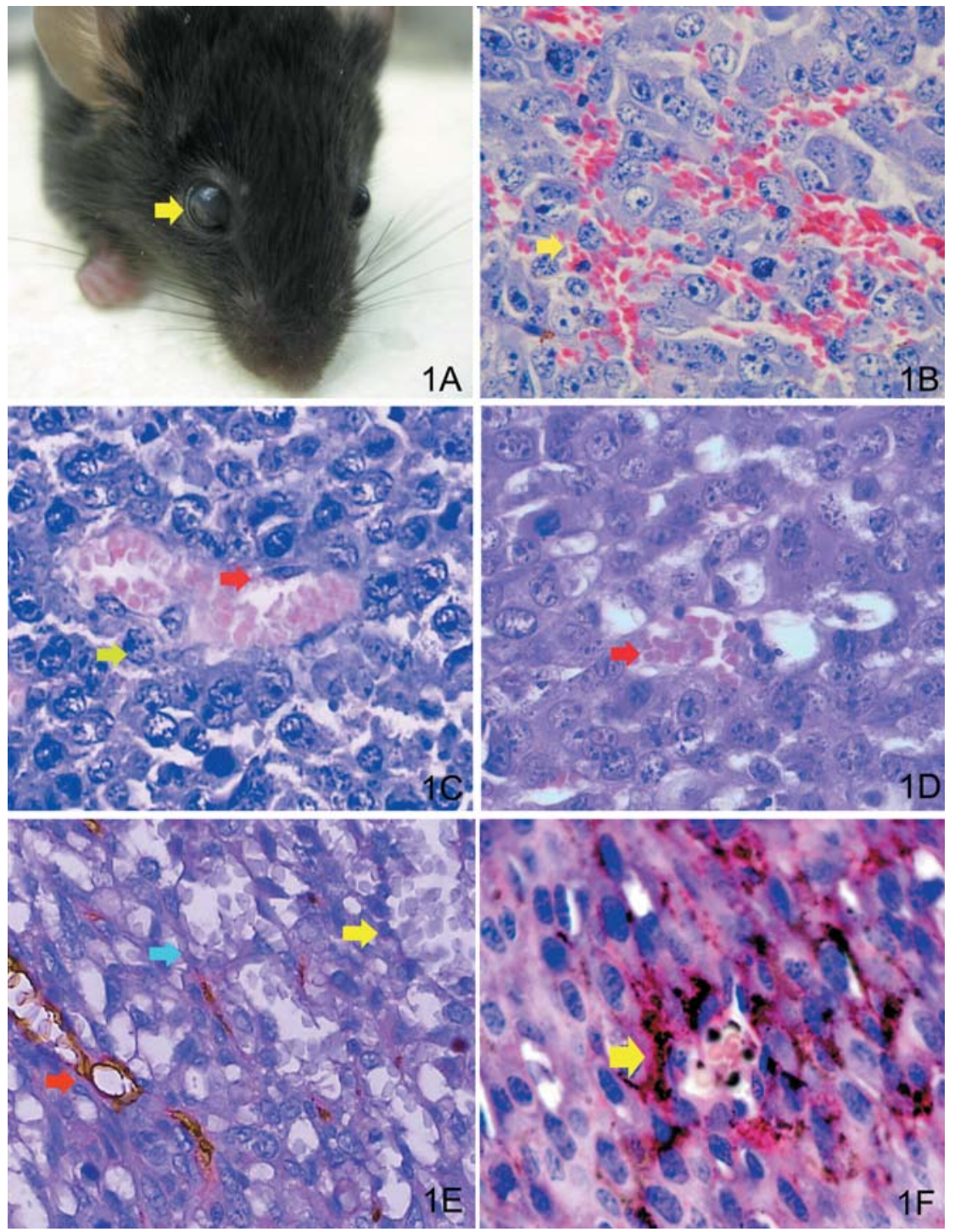

Figure 1. (A) A C57Bl/6 mouse carrying B16 melanoma, with pronounced proptosis of the right eye (yellow arrow, tumor). (B) Vasculogenic mimicry. It could be observed that encircled tumor cells form a tube structure containing red blood cells. Necrosis, inflammatory cells and red cell leakage were not found (yellow arrow, tumor) (H\&E, x400). (C) Mosaic vessel. Tumor cells (yellow arrow) and endothelial cells (red arrow) formed the mosaic vessel with red blood cells in it (H\&E, x400). (D) The endothelium-dependent vessels also could be observed in intraocular melanoma model (red arrow) (H\&E, x200) (E) VM channel was positive for PAS staining and negative for CD34 staining in this section (yellow arrow), while the endothelium-dependent vessel was positive for both PAS and CD34 staining (red arrow). A mosaic vessel was formed with tumor cells and endothelial cells (blue arrow) in this section by immunohistochemical double-staining (IHC, x400). (F) Results of the tumors showed numerous red blood cells and many brown granules activated carbons in the center of the VM (H\&E, x400).

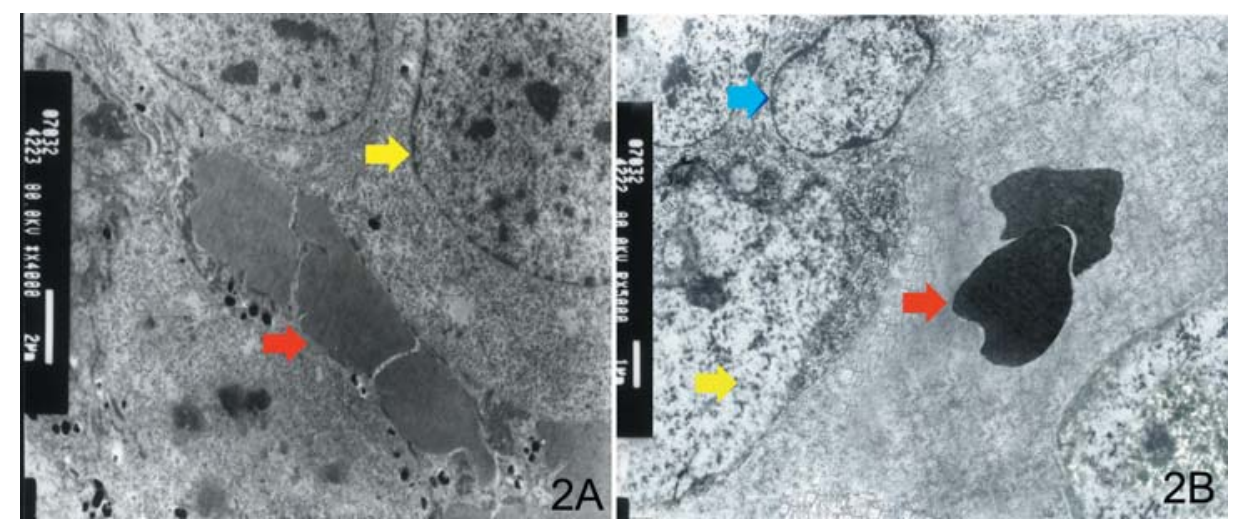

Figure 2. (A) This electron micrograph of VM channel showing its structure in melanoma model. Tumor cells (yellow arrow) form the tubular-like structure with a single-file column of RBC (red arrow) in it (x5000). (B) An electron micrograph showing the structure of a mosaic vessel in B16 model. Both tumor cells (yellow arrow) and endothelial cells (blue arrow) form the tube-like structure with RBC (red arrow) inside (x5000). 
a consequence of hemorrhage and necrosis of tumors, but many studies $(1,12,13)$ provided significant evidence to confirm it. A summary of the evidence is as follows: 1) Necrosis was not found around VM this indicated the tumor had an ample blood supply. Moreover, no endothelial cell-dependent vessels were found in the tumor while PAS-positive patterns were displayed. 2) Red blood cells were aligned in a single line in the PAS-positive pattern. 3) When capillaries grew into the tumor through sprouting, many red blood cells that leaked out of the vein could be seen in the space between the tumor cells. However, there were no leaking red blood cells around VM. Furthermore, Laser scanning confocal angiography, EM, and three-dimensional cell culture have confirmed the existence of VM.

Mueller et al reported that the OCM1 human uveal melanoma cells were injected into the eyes of SCID mice. This model that produced VM channels can be useful for tumor microcirculation mechanism (4). We think it is possible that different animal models will be useful for studying different physiological blood flow. In our previous study, it was reported that certain types of malignant tumors had MV. Nevertheless, our previously reported data indicated that as the tumor size grows and endothelial cells undergo continual mitosis, certain tumor cells of VM are replaced by endothelium (14). Additional study, however, is needed to elucidate the mechanism of VM. Reported theories about the mechanism include: 1) due to endothelial cells dropping from the vessel wall, tumor cells are directly exposed to the blood tube $(15) ; 2$ ) certain endothelial cells lose immunological marker activation in tumor progression, cannot be stained and become recessive cells; 3) tumor cells invade and are located in the blood vessel wall, and endothelial cells co-form the structure of blood vessels (16). Our studies of the ultramicrostructure of $\mathrm{VM}$ in malignant melanoma confirmed the results described above. One of our previous studies on the electron microscope showed that tumor cells lined the ECM and constructed tubular-like networks in malignant melanoma tissue, with the tubes containing some red blood cells $(17,18)$.

Microcirculation in this tumor not only provides enough nutrition and oxygen and disposes waste for tumor growth, but also provides the window and pathway for metastasis (19). Conventionally, vascular networks are believed to consist of tube structures lined with endothelial cells. In this study, there were three models in which tumors invaded the lungs of mice. The unique topology of VM distribution is consistent with the notion that specific molecular and cellular modifications occur to facilitate tumor invasion and growth. Therefore, VM is a poor prognosis indicator in patients with melanomas and other tumors (20-22). The three patterns of microcirculation developed by melanoma cells can supply blood and nutrients to the tumor. Endothelium-dependent vessels, which require the recruitment of normal endothelial cells, may not be efficient enough to sustain aggressive tumor growth at the early stage of rapid growth. Certain tumor cells, via dedifferentiation and changes in topology, connect with other tumor cells or endothelium and line the wall of the tube. Therefore, VM and MV become the sources of blood supply. They are just like co-workers for tumor tissue blood supply (23). Tumors that are more self-sufficient and can provide their own microcirculation components would have the greatest growth potential at the early stage of rapid growth. An interesting observation was that VM was most frequently observed in the boundary regions between the tumor and surrounding normal tissues (18). Thus, VM may also play an important role in tumor invasion by supplying immediate nutrition.

Our animal model inoculated with B16 cells suggested that the three above-mentioned blood supply patterns exist in the melanoma tissue with certainty. In the early stage of the first week the tumor requires nutrients and oxygen by permeation where there are no endothelial cells (19). With the tumor growing rapidly, endothelial cells from normal tissue around the tumor cannot meet the needs for tumor growth. Therefore, certain tumor cells connect with other tumor cells to line the wall of the tube via dedifferentiation and changes in topology, and VM becomes the major pattern of blood supply. It can be inferred that the three microcirculation patterns of blood supply including VM, MV and endotheliumdependent vessels may compose a series of stages in tumor growth. At the late stage, endothelium-dependent vessels are the major pattern of microcirculation.

Studies related to VM are still in an initial phase, and this novel blood supply pattern exists in many highly malignant tumors. Although it is not clear whether anti-cancer therapy targeted at endothelial cells has an effect on VM, the conception of VM poses a novel way to generate therapeutic strategies for some cancers. Inhibiting the expression of certain tumor-related adhesion molecules, controlling the synthesis of proteases such as EphA2, MMPS and laminin-5 $\gamma-2$ chain associated with tumor invasion, could control these highly malignant tumors with VM (24-26). This study showed that $\mathrm{VM}$ was observed when the average diameter of tumor was $\sim 4 \mathrm{~mm}$. However, Seftor et al (27) reported that VM formed only after the average size reached $10 \mathrm{~mm}$ in the subcutaneous melanoma model. Possibly, it is due to the small size of the mouse eye. As the tumor proliferates, the intraocular pressure (IOP) increases greatly. The secondary-glaucoma is usually associated with elevated IOP and may occur at late manifestation. Tumor cells in a high-pressure microenvironment must secrete proteinases to enable continual growth, invasiveness and metastasis. Hence, high interstitial fluid pressure has more influence on tumor invasiveness and microcirculation patterns than the other factors (29). High microenvironment pressure was one of the main factors that lead to the formation of VM in melanomas. When a tumor mass is ischemic and hypoxic, tumor cells will change their blood supply patterns.

Clinical data also showed that nearly all patients with highgrade malignant melanoma die of tumor metastasis via the bloodstream (30-32). Therapy that only targets at angiogenesis may not have distinguished effects. Therefore, the existence of an alternative microcirculation plays an important role in anti-angiogenesis treatment, and additional forms of therapy are necessary. In this case, VM is the main blood supply with high intraocular pressure while the endothelium-dependent vessel is the main microcirculation pattern with low pressure in a blood supply. As a result, the intraocular melanoma tended to metastasize and seemed to be more aggressive than previous shown in subcutaneous melanoma. The adoption of VM and MV may be a way to adapt to rapid tumor growth and metastasis. Therefore, we should adopt the concept of 
therapy targets not only on anti-angiogenesis, but also on anti-VM and anti-MV. Further research on tumor cell plasticity in melanoma is necessary.

\section{Acknowledgements}

This work was partially supported by the National Science Foundation of China.

\section{References}

1. Maniotis AJ, Folberg R, Hess A, et al: Vascular channel formation by human melanoma cells in vivo and in vitro: vasculogenic mimicry. Am J Pathol 155: 739-752, 1999.

2. Folberg R, Hendrix MJC and Maniotis AJ: Vasculogenic mimicry and tumor angiogenesis. Am J Pathol 156: 361-381, 2000.

3. McDonald DM, Munn L and Jain RK: Vasculogenic mimicry: how convincing, how novel, and how significance? Am J Pathol 156: 383-387, 2000 .

4. Mueller AJ, Maniotis AJ, Freeman WR, Bartsch D-U, Schaller UC, Bergeron-Lynn G, et al: An orthotopic model for human uveal melanoma in SCID mice. Microvasc Res 64: 207-213, 2002.

5. Zhang S, Zhang D, Wang Y, Zhao W, Guo H, Zhao X, et al: Morphologic research of microcirculation patterns in human and animal melanoma. Med Oncol 23: 3403-3409, 2006.

6. Grossniklaus HE, Dithmar S and Albert DM: Animal models of uveal melanoma. Mel Res 10: 195-211, 2000.

7. Ma D, Gerard R, Li X, Alizadeh H and Niederkorn J: Inhibition of metastasis of intraocular melanomas by adenovirus-mediated gene transfer of plasminogen activator inhibitortype I (PAI-1) in an athymic mouse model. Blood 90: 2738-2746, 1997.

8. Ma D and Niederkorn J: Role of epidermal growth factor receptor in the metastasis of intraocular melanomas. Invest Ophthalmol Vis Sci 39: 1067-1075, 1998

9. Clarijs R, Otte-Hoåller I, Ruiter DJ and de Waal RMW: Presence of a fluid-conducting meshwork in xenografted cutaneous and primary human uveal melanoma. Invest Ophthalmol Vis Sci 43: 912-918, 2002

10. Rummelt V, Mehaffey MG, Campbell RJ, Péer J, Bentler SE, Woolson RF, Naumann GO and Folberg R: Microcirculation architecture of metastases from prime ciliary body and choroidal melanomas. Am J Ophthalmol 126: 303-305, 1998.

11. Sharma N, Seftor RE, Seftor EA, Gruman LM, Heidger PM Jr, Cohen MB, et al: Prostatic tumor cell plasticity involves cooperative interactions of distinct phenotypic subpopulations: role in vasculogenic mimicry. Prostate 50: 189-201, 2002.

12. Sun B, Zhang D, Zhang S, Zhang W, Guo H and Zhao X: Hypoxia influences vasculogenic mimicry channel formation and tumor invasion-related protein expression in melanoma. Cancer Lett 249: 188-197, 2007.

13. Sood AK, Fletcher MS, Coffin JE, Yang M, Seftor EA, Gruman LM, et al: Functional role of matrix metalloproteinases in ovarian tumor cell plasticity. Am J Obstet Gynecol 190: 899-909, 2004

14. Zhang S, Hua G, Zhang D, Zhang W, Zhao X, Ren Y, et al: Microcirculation patterns in different stages of melanoma growth. Oncol Rep 15: 15-20, 2006.
15. Folberg R and Maniotis AJ: Vasculogenic mimicry. APMIS 112: 508-525, 2004.

16. Stephenson J: Mosaic vessels shed cancer clues by the million. Lancet Oncol 2: 130, 2001.

17. Drewa G, Wozqak A, Palgan K, Schacht DO, Grzanka A and Sujkowska R: Influence of quercetin on B16 melanotic melanoma growth in C57Bl/6 mice and on activity of some acid hydrolases in melanoma tissue. Neoplasma 48: 12-18, 2001.

18. Sun B, Zhang S, Zhao X, Zhang W and Hao X: Vasculogenic mimicry is associated with poor survival in patients with mesothelial sarcomas and alveolar rhabdomyosarcomas. Int $\mathbf{J}$ Oncol 25: 1609-1614, 2004.

19. Hendrix MJ, Seftor EA and Hess AR: Vasculogenic mimicry and tumour-cell plasticity: lessons from melanoma. Nat Rev Cancer 3: 411-421, 2003.

20. Ilmonen S, Kariniemi AL, Vlaykova T, Muhonen T, Pyrhonen S and Asko-Seljavaara S: Prognostic value of tumor vascularity in primary melanoma. Melanoma Res 9: 273-242, 1999.

21. Shirakawa K, Kobayashi H, Sobajima J, Hashimoto D, Shimizu A and Wakasugi $\mathrm{H}$ : Inflammatory breast cancer: vasculogenic mimicry and its hemodynamics in an inflammatory breast cancer xenograft model. Breast Cancer Res 5: 136-139, 2003.

22. Hendrix MJ, Seftor EA, Hess AR and Seftor RE: Molecular plasticity of human melanoma cells. Oncogene 22: 3070-3075, 2003.

23. Hendrix MJ, Seftor EA, Kirschmann DA, Quaranta V and Seftor RE: Remodeling of the microenvironment by aggressive melanoma tumor cells. Ann NY Acad Sci 995: 151-161, 2003.

24. Vartanian AA, Burova OS, Stepanova EV, Baryshnikov AY and Lichinitser MR: Melanoma vasculogenic mimicry is strongly related to reactive oxygen species level. Melanoma Res 17: 370-379, 2007.

25. Schaft DW, Seftor RE, Seftor EA, Hess AR, Gruman LM, Kirschmann DA, et al: Effects of angiogenesis inhibitors on vascular network formation by human endothelial and melanoma cells. J Natl Cancer Inst 96: 1473-1477, 2004.

26. Hess AR, Seftor EA, Gruman LM, Kinch MS, Seftor RE and Hendrix MJ: VE-cadherin regulates EphA2 in aggressive melanoma cells through a novel signaling pathway: implications for vasculogenic mimicry. Cancer Biol Ther 5: 228-233, 2006.

27. Seftor RE, Seftor EA, Kirschmann DA and Hendrix MJ: Targeting the tumor microenvironment with chemically modified tetracyclines: inhibition of laminin 5 gamma 2 chain promigratory fragments and vasculogenic mimicry. Mol Cancer Ther 1: 1173-1179, 2002.

28. Sun B, Zhang S, Zhang D, Gu Y, Zhang W and Zhao X: The influence of different microenvironments on melanoma invasiveness and microcirculation patterns: an animal experiment study in the mouse model. J Cancer Res Clin Oncol 133: 979-985, 2007.

29. Pietras K, Ostman A, Sjoquist M, Buchdunger E, Reed RK, Heldin $\mathrm{CH}$, et al: Inhibition of platelet-derived growth factor receptors reduces interstitial hypertension and increases transcapillary transport in tumors. Cancer Res 61: 2929-2934, 2001.

30. Saif MV: Anti-angiogenesis therapy in pancreatic carcinoma. JOP 7: 163-173, 2006.

31. Hillen F and Griffioen AW: Tumour vascularization: sprouting angiogenesis and beyond. Cancer Metastasis Rev 26: 489-502, 2007.

32. Schachter J, Katz U, Mahrer A, Barak D, David LZ, Nusbacher J, et al: Efficacy and safety of intravenous immunoglobulin in patients with metastatic melanoma. Ann NY Acad Sci 1110: 305-314, 2007. 